\title{
Segmental Hypoganglionosis of the Colon: A Case Report
}

\author{
Aya Yamagishi ${ }^{1}$, Nobuatsu Koyama ${ }^{1}$, Naoyuki Yamashita ${ }^{1}$, Mikito Suzuki ${ }^{1}$, \\ Takeshi Yamada ${ }^{2}$, Manpei Kawashima ${ }^{2}$ and Hiroshi Yoshida ${ }^{2}$ \\ ${ }^{1}$ Department of Surgery, Tsuboi Hospital, Koriyama City, Japan \\ ${ }^{2}$ Department of Gastrointestinal and Hepato-Biliary-Pancreatic Surgery, Nippon Medical School, Tokyo, Japan
}

\begin{abstract}
A 64-year-old woman was admitted to hospital with persistent abdominal pain. She had been hospitalized with similar symptoms on five occasions during a period of 2 years. Computed tomography revealed dilatation and fecal impaction from the ileum to the transverse colon. A barium enema and simultaneous ileus tube radiography showed a narrow segment of descending-sigmoid colon. Colonoscopy showed no mucosal change. Her symptoms did not improve with conservative therapy, so descending and sigmoid colectomy was performed. Histologic examination showed disappearance of ganglion cells; axon of Meissner's plexuses was present, and the number of Auerbach's plexuses was decreased. The definitive diagnosis was segmental hypoganglionosis $(\mathrm{SH})$ of the colon. The postoperative course was uneventful, and the functional result was positive at 1 year postoperatively. $\mathrm{SH}$ is extremely rare; however, surgical intervention is expected to be of benefit. Therefore, it is important to keep SH in mind when treating patients with chronic obstruction of the left side of the colon.
\end{abstract}

(J Nippon Med Sch 2021; 88: 370-374)

Key words: colectomy, colon, dilatation

\section{Introduction}

Segmental hypoganglionosis $(\mathrm{SH})$ is a rare condition. One of the main symptoms of $\mathrm{SH}$ is chronic intermittent obstruction caused by partial expansion failure of the intestine, wherein the numbers of neural plexuses and ganglion cells are decreased in the submucosal or intramucosal layers $^{1,2}$. SH is sometimes regarded as a subtype of colonic pseudo-obstruction or as a disease similar to adult Hirschsprung disease, but the term is controversial. Because definitive diagnosis is only possible by determining the histochemical characteristics of a fullthickness biopsy specimen, it can be difficult to diagnose preoperatively. This study reports a surgical case of $\mathrm{SH}$ in which the patient had longstanding recurrent obstruction symptoms.

\section{Case Report}

A 64-year-old woman was admitted to hospital for assessment of severe abdominal pain and constipation during the previous few days. She had been prone to consti- pation for several years, occasionally used laxatives, and had previously been admitted to hospital with similar symptoms five times in the past 2 years. Her medical and family histories were unremarkable. Physical examination showed gross abdominal distention, moderate tenderness in the epigastrium, and no rebound tenderness. Bowel sounds were active and showed no increase. Laboratory findings, including a white blood cell count and C-reactive protein, were normal. An abdominal X-ray showed the multiple air-fluid level of the ileum and colon dilatation across the entire abdomen. Abdominal computed tomography (CT) revealed dilatation with feces present from the ileum to the transverse colon. The dilated transverse colon was elongated, but there was no significant obstruction and no sign of tumor lesions (Fig. 1). Colonoscopy revealed a narrow segment in the descending colon and sigmoid colon with intact mucosa (Fig. 2). Ileus tube radiography and barium enema were performed simultaneously and revealed a narrow segment in the descending colon and sigmoid colon with

Correspondence to Aya Yamagishi, MD, PhD, Department of Surgery, Tsuboi Hospital, 1-10-13 Nagakubo, Asakamachi, Koriyama City, Fukushima 963-0197, Japan

E-mail: ayatani@nms.ac.jp

https://doi.org/10.1272/jnms.JNMS.2021_88-413

Journal Website (https://www.nms.ac.jp/sh/jnms/) 


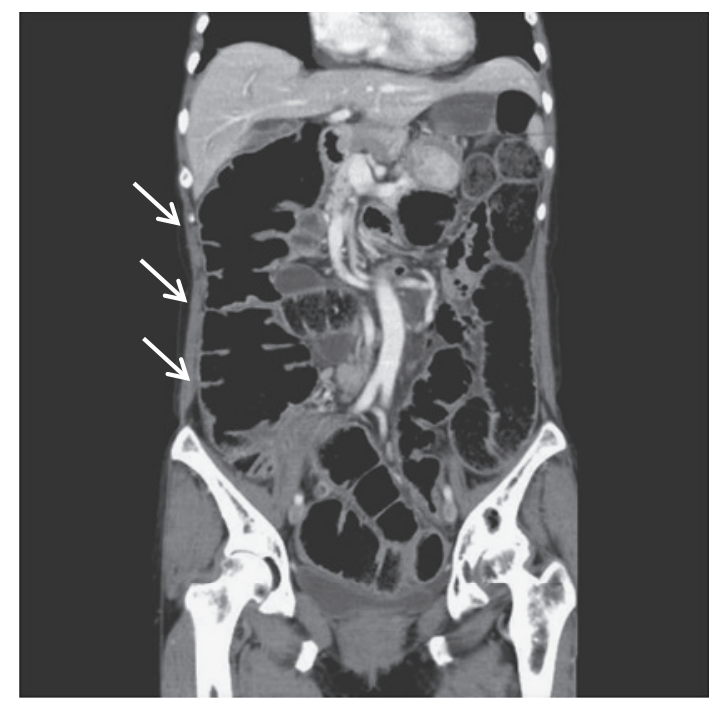

Fig. 1 Abdominal computed tomography (CT) Abdominal CT scan revealed dilatation and elongation of the ascending to the transverse colon. Residue build-up and dilation of the ileum were also observed.

spastic change and diverticula and a dilated colon and ileum above the stricture (Fig. 3). Conservative therapy with fasting and ileus tube radiography was effective; however, surgery was proposed owing to the patient's recurrent symptoms. The patient and her family provided informed consent.

Intraoperatively, we noted a significantly dilated and redundant ascending and transverse colon (Fig. 4). The descending and sigmoid colon were both spastic and hard, the intestinal diameter was remarkably small, and diverticulitis was present. This area was considered to be responsible for the disease, so it was decided that the lesion between the middle of the transverse colon and sigmoid colon would be removed. We performed primary anastomosis by functional end-to-end anastomosis. Because of the considerable risk of leakage from the dilated intestine, we added a buried suture to staple line and performed decompression by ileus tube radiography perioperatively, to further prevent anastomotic leakage. The transverse colon was markedly dilatated, to $14 \mathrm{~cm}$ in diameter, while the descending colon was $4.5 \mathrm{~cm}$, and there was no tumor lesion in the mucosa (Fig. 5a). Histologic examination revealed no ganglion cells or fibers in Meissner's plexuses, and the number of Auerbach's plexuses was diminished, with a degenerative tendency of the ganglion cells in the narrow segment (Fig. 5b-e). The definitive diagnosis was $\mathrm{SH}$ of the colon. The postoperative period was uneventful, and the patient was discharged 14 days postoperatively. Follow-up information was obtained at 1 year postoperatively in the outpatient clinic,

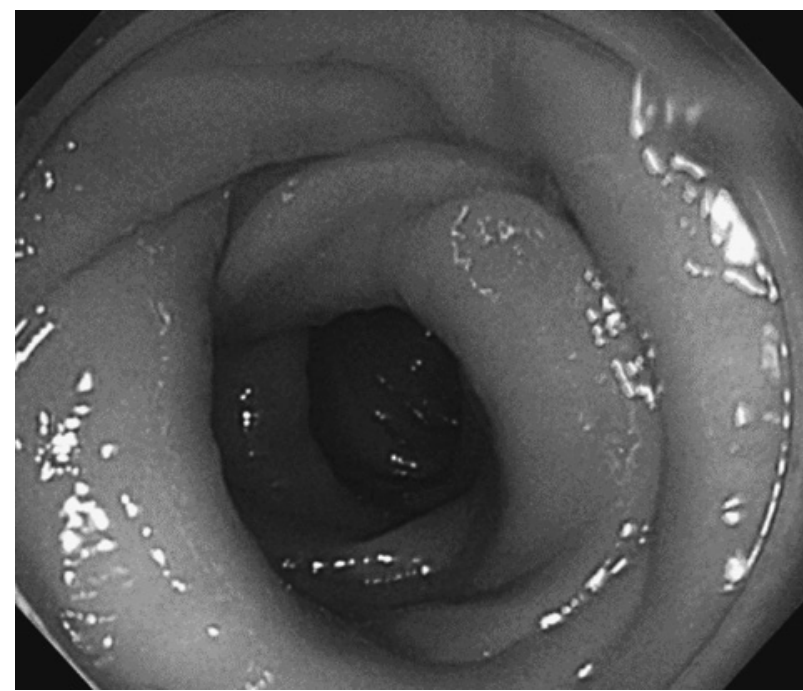

Fig. 2 Lower gastrointestinal endoscopy findings Colonoscopy showed a narrow segment in the descending colon and sigmoid colon with intact mucosa.

and the patient was well and normally continent.

\section{Discussion}

SH has been reported in patients with segmental dilation failure, diminution of ganglion cells, and degeneration of Auerbach's or Meissner's plexuses in a segment with poor dilation on histopathologic findings ${ }^{1}$. There is no established theory regarding the cause of this condition, although degeneration and disappearance of myenteric neurons secondary to anoxemia, inflammation, and/or viral infection of the bowel wall are involved ${ }^{2-4}$.

Intramural neurons of the intestinal tract develop from vagus nerves in the early fetal stage, during which neuroblasts migrate down the gastrointestinal tract sequentially in a rostral to caudal manner, as proposed by the craniocaudal migration theory ${ }^{5}$. Abnormal development during this stage can cause esophageal achalasia, which is characterized by abnormalities of gangliocytes in the lower esophagus, while congenital absence of neurons of the intestinal wall continuously from the rectum is known as Hirschsprung disease.

$\mathrm{SH}$ is acquired, subacute, and relatively common in adults. Lesions are observed in the segment between the descending colon and sigmoid colon. Mecholyl, a cholinergic agent previously used for diagnostic tests, provokes a hyper-reaction to the autonomic drug at the segment with the neural defect but is no longer used because it causes adverse reactions in the circulatory system ${ }^{6}$. Specific markers, such as radiopaque markers, may be useful for assessing transition time in functional evaluation. $\mathrm{SH}$ can only be diagnosed by histochemical examination of a 


\section{A. Yamagishi, et al}
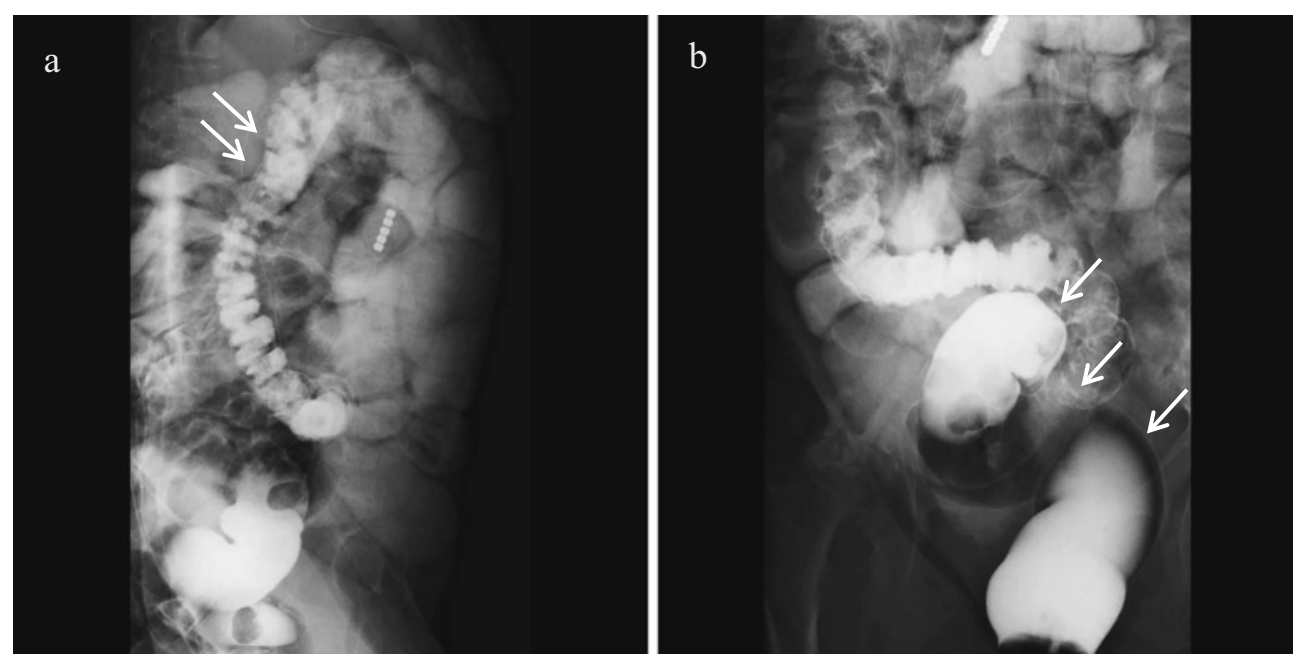

Fig. 3 Ileus tube radiography and barium enema.

Ileus tube radiography revealed no obstructive lesion in the ileum or dilated ascending colon.

a. A barium enema study clearly revealed the transition zone of the dilated transverse to the narrow descending colon.

b. The rectum was dilated by insufflation.

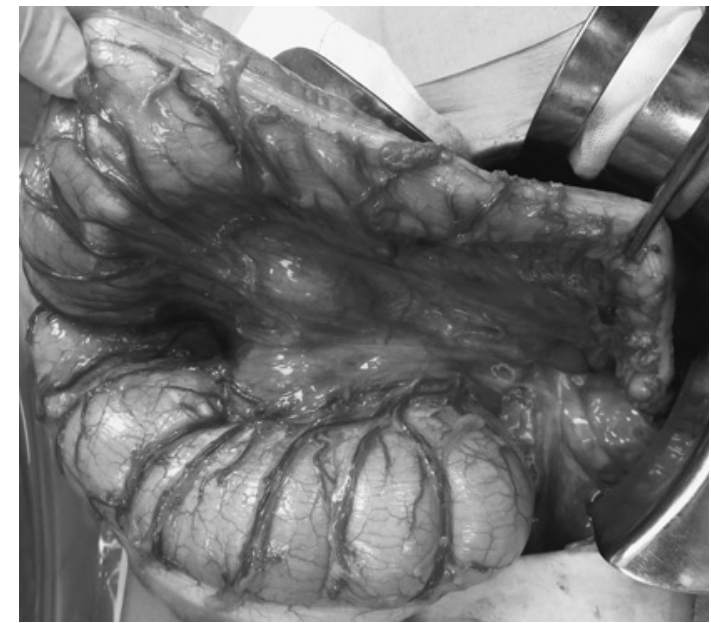

Fig. 4 Surgical findings

Surgery revealed that the descending and sigmoid colon were narrow, with spastic change and diverticula. The ascending and transverse colon were redundant and dilated.

full-thickness biopsy specimen. Histopathologic characteristics include a significant reduction in the number of ganglion cells, thickened muscularis mucosae, and muscularis propria layers ${ }^{7}$. More recently, S-100 and peripherin were validated as valuable tools for diagnosis of $\mathrm{SH}^{8}$. The numbers of c-kit-immunoreactive cells and 171B5 (a novel marker of synapses)-immunoreactive synapses were diminished in muscle layers of bowel affected by Allied Hirschsprung disease. Infiltration of T lymphocytes can lead to the death of neurons and thus a reduction in c-kit signaling ${ }^{10}$. Double-contrast barium enema and CT images reveal a caliber change, as compared with the dilated proximal colonic segment to narrow distal segment.

Surgery is considered the definitive treatment for $\mathrm{SH}^{11}$. The stricture site alone may be resected ${ }^{7,12}$, but some cases require subtotal proctocolectomy for marked dilatation or when extensive resection is deemed necessary to remove the dilated section. An inadequately demarcated resection site can result in anastomotic stricture.

A number of medical conditions can cause recurrent bowel obstruction ${ }^{13}$. Dudley et al. described the clinicopathologic condition of chronic functional obstruction without organic obstruction as chronic intestinal pseudoobstruction $(\mathrm{CIP})^{14}$. CIP can be classified according to the affected areas: CIP limited to the colon is defined as a colonic pseudo-obstruction $(\mathrm{CPO})^{15}$. Primary $\mathrm{CPOs}$ are caused by digestive tract lesions and secondary CPO by systemic disease or drugs; some CPOs are idiopathic. Primary CPO comprises Hirschsprung disease and Allied disorders of Hirschsprung disease, including $\mathrm{SH}^{1,6}$. Secondary CPO has drawn attention in recent studies of the involvement of viral infections. Debinski et al., for example, used nested polymerase chain reaction to test for the presence of EBV and CMV in $\mathrm{CPO}^{16}$.

Hirschsprung disease is marked by dysperistalsis and absence of an anorectal reflex originating in aganglionosis of the distal enteric tract. It can result in impaired passage of intestinal contents, delayed meconium passing, and megacolon. Most cases are congenital, but chronic Hirschsprung disease of adulthood has been reported $^{17}$. A group of diseases with symptoms and test 


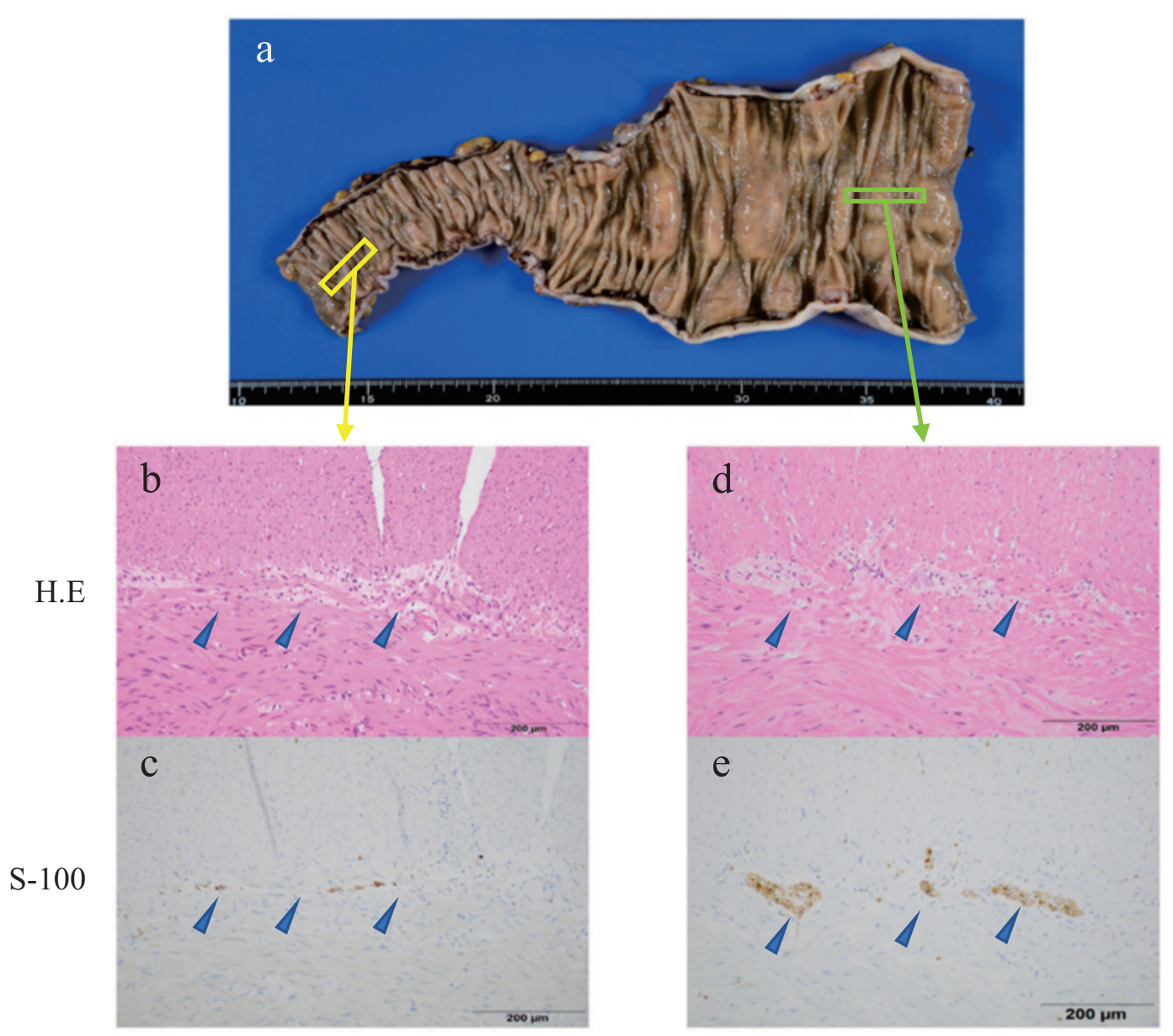

Fig. 5

a. Macroscopic appearance of marked caliber change and intact mucosa.

$\mathrm{b}$ and c. Histopathological findings for tissue shown in the yellow square of segmental hypoganglionosis in a.

$\mathrm{d}$ and e. Histopathological findings for tissue shown in the green square of the normal area in a.

Fewer ganglion cells (arrowheads) were present than in the normal area (b, d: hematoxylin and eosin stain; c, e: S-100 stain).

findings similar to those of Hirschsprung disease, despite the presence of normal ganglion cells in the rectum, has recently been reported in Japan and termed Allied Hirschsprung diseases ${ }^{18}$. Most patients develop such diseases during childhood, but onset can occur in adulthood, as in the case of $\mathrm{SH}$.

Several studies related to $\mathrm{SH}$ were reviewed, and 17 cases were reported in Japan. Patient age was 31 to 72 years, and the male:female ratio was 11:5. The site of the disease was localized from the transverse to descending colon in all patients. All patients were treated surgically, except for two patients who underwent subtotal colectomy. Surgery involved resection of the affected segment of the colon. However, there is no consensus on how the extent of resection should be determined.

Because of the lack of clear diagnostic criteria for $\mathrm{SH}$, the present case of $\mathrm{SH}$ was diagnosed by general assess- ment of symptom course, laboratory findings, and diagnostic surgical pathology. Preoperatively, SH was not considered the primary disease. The stricture site was established as the extent of resection on the basis of intraoperative findings suggesting subobstruction due to stagnation and volvulus on the rostral side of the narrowed enteric tract. The anal side of the tract was long and thus reconstructed by functional end-to-end anastomosis, which improved defecation symptoms without postoperative stricture of the anastomotic site. Functional end-to-end anastomosis is a useful method because of the low frequency of bleeding, stenosis, and anastomotic leakage ${ }^{19}$. Blind loop syndrome is a unique complication of functional anastomosis. For this disease, in intestinal tract with neural abnormalities, the anastomosis method requires more attention and follow-up, and an obtuse angle must be devised for the intestinal tract. The extent of 


\section{A. Yamagishi, et al}

resection was deemed adequate; however, histopathological findings showed degeneration of ganglion cells on the anal margin, and the healthy segment was not included. There have been similar cases in which only the dilated segment was resected because $\mathrm{SH}$ was not considered to be the cause preoperatively. This also included cases in which the main lesion was not completely resected, resulting in postoperative recurrent stricture, and cases in which the anal margin included the lesion, thus causing anastomotic stricture. Rapid pathological diagnosis was difficult because ganglion cells were disseminated and examination of ganglion cells in the long axis of the intestine was necessary. We thus secured a centimeter margin from the stricture site. Detailed pathological examination of the resected specimen is necessary for assessing neural abnormality in $\mathrm{SH}$; thus, it is important to pay attention to clinical features such as recurrent bowel obstruction without organic obstruction and laboratory findings, to include $\mathrm{SH}$ in the preoperative differential diagnosis, and to plan therapeutic strategies accordingly. To establish diagnostic criteria for $\mathrm{SH}$, future cases such as these should be investigated.

We reported a case of $\mathrm{SH}$ in which surgical treatment after a long symptomatic period allowed for histopathological diagnosis.

Acknowledgments: We authors are grateful for the pathology support provided by Dr. Seiji Igarashi and Dr. Miho Kobayashi (Department of Pathology, Tsuboi Hospital) and for the translation and editing support provided by Crimson Interactive Pvt. Ltd. (Ulatus) - www.ulatus.jp.

Conflict of Interest: The authors declare no conflicts of interest.

\section{References}

1. Matsui T, Iwashita A, Iida M, Kume K, Fujishima M. Acquired pseudoobstruction of the colon due to segmental hypoganglionosis. Gastrointest Radiol. 1987;12(3):262-4.

2. Touloukian RJ. Acquired aganglionic megacolon in a premature infant: report of a case. Pediatrics. 1975;56(3):45962.

3. Sprinz H, Cohen A, Heaton LD. Hirschsprung's disease with skip area. Ann Surg. 1961;153(1):143-8.

4. Holland-Cunz S, Göppl M, Rauch U, Bär C, Klotz M, Schäfer KH. Acquired intestinal aganglionosis after a lytic infection with varicella-zoster virus. J Pediatr Surg. 2006; 41(3):e29-31.

5. Fujimoto T, Hata J, Yokoyama S, Mitomi T. A study of the extracellular matrix protein as the migration pathway of neural crest cells in the gut: analysis in human embryos with special reference to the pathogenesis of Hirschsprung's disease. J Pediatr Surg. 1989;24(6):550-6.

6. Suzuki H, Amano S, Matsumoto K, Kitagawa T, Masuda T. Chronic idiopathic intestinal pseudo-obstruction caused by acquired visceral neuropathy localised in the left colon: report of two cases. Jpn J Surg. 1987;17(4):302-6.

7. Pan $\mathrm{ZP}$, Huang LQ, Cui JH. Acquired segmental sigmoid hypoganglionosis: A case report. Med (Baltim). 2020;99(4): e18803.

8. Holland SK, Hessler RB, Reid-Nicholson MD, Ramalingam P, Lee JR. Utilization of peripherin and S-100 immunohistochemistry in the diagnosis of Hirschsprung disease. Mod Pathol. 2010;23(9):1173-9.

9. Yamataka A, Ohshiro K, Kobayashi H, Fujiwara T, Sunagawa M, Miyano T. Intestinal pacemaker C-KIT+ cells and synapses in allied Hirschsprung's disorders. J Pediatr Surg. 1997;32(7):1069-74.

10. Faussone-Pellegrini MS, Fociani P, Buffa R, Basilisco G. Loss of interstitial cells and a fibromuscular layer on the luminal side of the colonic circular muscle presenting as megacolon in an adult patient. Gut. 1999;45(5):775-9.

11. Qadir I, Salick MM, Barakzai A, Zafar H. Isolated adult hypoganglionosis presenting as sigmoid volvulus: a case report. J Med Case Rep. 2011;5:445.

12. Poisson J, Devroede G. Severe chronic constipation as a surgical problem. Surg Clin North Am. 1983;63(1):193217.

13. Miyao M, Takahashi T, Okusa M, et al. Recurrent bowel obstruction cased by cecal volvulus: a case report. J Nippon Med Sch. 2019;86(3):183-6.

14. Dudley HA, Sinclair IS, McLaren IF, McNair TJ, Newsam JE. Intestinal pseudo-obstruction. J R Coll Surg Edinb. 1958;3(3):206-17.

15. Anuras S, CR Baker Jr. The colon in the pseudoobstructive syndrome. Clin Gastroenterol. 1986;15(4):745-62.

16. Debinski HS, Kamm MA, Talbot IC, Khan G, Kangro HO, Jeffries DJ. DNA viruses in the pathogenesis of sporadic chronic idiopathic intestinal pseudo-obstruction. Gut. 1997;41(1):100-6.

17. Miyamoto M, Egami K, Maeda S, et al. Hirschsprung's disease in adults: report of a case and review of the literature. J Nippon Med Sch. 2005;72(2):113-20.

18. Muto M, Matsufuji $H$, Taguchi T, et al. Japanese clinical practice guidelines for allied disorders of Hirschsprung's disease, 2017. Pediatr Int. 2018;60(5):400-10.

19. Steichen FM. The use of staplers in anatomical side-toside and functional end-to-end enteroanastomoses. Surgery. 1968;64:948-53.

(Received, June 2, 2020)

(Accepted, September 11, 2020)

(J-STAGE Advance Publication, November 30, 2020)

Journal of Nippon Medical School has adopted the Creative Commons Attribution-NonCommercial-NoDerivatives 4.0 International License (https://creativecommons.org/licenses/by-nc-nd/4.0/) for this article. The Medical Association of Nippon Medical School remains the copyright holder of all articles. Anyone may download, reuse, copy, reprint, or distribute articles for non-profit purposes under this license, on condition that the authors of the articles are properly credited. 